\title{
LE PARADIGME ESTHÉTIQUE : LA SOCIOLOGIE COMME ART
}

\author{
Michel Maffesoli ${ }^{1}$ \\ «Les originaux ont généralement été des nommeurs. » \\ (F.NIETZSCHE, le Gai savoir)
}

\section{I - L'ESSAI SAVANT}

À l'encontre du «social engineering», sous ses formes plus ou moins sophistiquées, peut-être est-il temps de réclamer pour le sociologue le droit de poétiser, d'esthétiser sur le développement social. C'est-à-dire être au plus près, sentir ce développement qui, dans le sens que lui donnait le romantisme allemand («entwicklung »), est plus proche de la croissance que de l'évolution.

On recommence à savoir, et surtout à ne plus avoir peur de dire, que la connaissance ne se limite pas à la science : à tout le moins à une certaine forme de la science. Ainsi être attentif à ce que j'ai proposé d'appeler la « respiration sociale » ne sera pas attitude de circonstance, mais une exigence intellectuelle qui sache intégrer la multiplicité des éléments composant cette société de plus en plus complexe qui est en train de se constituer. Il est d'ailleurs intéressant de noter que l'on retrouve sous la plume de E. Canetti des références à la «mémoire respiratoire » ou à «l'économie respiratoire ». Il note que quoique la science - ce «parvenu de l'humanité » dit-il - ait négligé cette dimension, l'organisation de nos sociétés, de nos cités, repose sur l'ajustement des «espaces de respiration ${ }^{2} »$. En rappelant que la mythologie véhicule nombre d'images atmosphériques, on soulignera que de tout temps le souffle et la vie sont intimement liés. Signalons enfin que les techniques respiratoires qui nous viennent de l'Orient s'inscrivent à n'en pas douter dans un processus de connaissance que l'on ne peut plus négliger.

Ces références cavalières permettent de faire ressortir l'importance de l'ambiance qui caractérise une époque. Dès lors au-delà de la réduction conceptuelle, la description, la saisie d'impressions, toutes choses mélangeant la phénoménologie et la poésie, pourront trouver leurs places dans nos disciplines. La multiplicité des respirations sociales, autre manière de dire styles ou modes de vie, n'est pas la simple conséquence de tel ou tel facteur économique ou politique, ne peut pas non plus se

\footnotetext{
${ }^{1}$ Paris V, Sorbonne, França.

${ }^{2}$ E. Canetti, la Conscience des mots, Paris, Albin Michel, 1984, p.24 sq.
} 
résumer dans une simple catégorisation psychologique. Elle a sa propre autonomie, et traduit en quelque sorte une vie animale, irrépressible et quelque peu barbare ; mixte de passions et de sentiments, vie qui a de plus en plus tendance à faire éclater l'aspect policé de la civilisation.

C'est cela qui peut nous inciter à dépasser la vieille méfiance qui, depuis Platon, pèse sur la «jouissance », y compris la jouissance intellectuelle. On se souvient par exemple comment, au début de l'ère bourgeoise, Faust va détrôner le Verbe et écrire «tout simplement: Au commencement était l'action!» (Goethe, Faust I, Cabinet d'Étude). C'est dans cette lignée que de leurs cabinets d'étude également les intellectuels vont voir dans les diverses modulations de l'action les seuls vecteurs de la vie sociale.

Il faudra y revenir, mais il semblerait que le devenir communicationnel de nos sociétés nous oblige à reconsidérer le problème : accorder à nouveau au Verbe la place qui lui revient, et faire attention aux images et à la rhétorique sociale qui du Café du commerce aux discours politiques ponctuent l'existence quotidienne. Le mot, celui de la rencontre ou celui de la messagerie informatique, est un élément interactif et comme tel mérite crédit. Dès lors le jeu ou la jouissance du mot du sociologue ne sera pas un simple «flatus vocis », mais bien expression de l'ambiance d'un moment.

En un temps qui n'est pas sans rappeler le notre, celui de l'antiquité tardive, face aux philosophes se délectant dans l'abstraction, Saint-Augustin sut manier, avec bonheur, la méthode allégorique. Ce faisant il prenait acte de l'écroulement d'un monde, et sa parole n'est pas pour rien dans la constitution de la civilisation chrétienne qui s'élaborait. La prolifération d'images de ses analyses est en parfaite consonnance avec l'éclatement du réel qu'il observait ${ }^{3}$. Ce pour dire que toute chose nouvelle qui émerge a besoin de visualiser son objectif et par là même de se visualiser. Le développement technologique en plus, on peut faire le rapprochement entre la fin du monde antique et la fin de la modernité : aux images immatérielles de la post-modernité doit pouvoir correspondre la démarche allégorique de nos analyses. On peut ici faire référence au très beau livre de R. A. Nisbet qui, en particulier lorsqu'il s'interroge sur l'imagination sociologique des pères fondateurs, ne manque pas de souligner qu'ils « ne cessèrent jamais [...] d'être des artistes ». Pour lui il est possible d'envisager la «sociology as an Art Form». Ainsi les concepts que nous utilisons résulteraient sans

\footnotetext{
${ }^{3}$ Sur l'allégorie, cf. l'historien P.Brown, la Vie de saint Augustin, Paris, Seuil, 1971, p. 308 et note 13.
} 
exception de ces intuitions ou visions qui sont «tout autant propres à l'artiste qu'au savant $^{4} \gg$. Les exemples qu'il donne en ce sens sont tout à fait probants et montrent bien l'inanité des définitions préliminaires dans la démarche scientifique. Il signale également que Simmel est «celui des grands sociologues chez lequel l'imagination et l'intuition jouent le plus grand rôle $»^{5}$. D'une manière plus précise d'ailleurs, au travers de l'exemple de Simmel ce qui est indiqué c'est que toute grande œuvre repose sur la tension paradoxale d'aspects esthétiques et d'aspects généraux. C'est ce qui fait la richesse de ces œuvres et surtout ce qui les différencie « de certaines des sciences physiques »; j'ajouterai ce qui les différencie de toutes celles de ces ingénieurs sociaux qui on décrété une fois pour toute que la scientificité se mesurait à l'aune de l'ennui qu'elles suscitent.

En fait le non-conformisme dans la démarche simmelienne, sa prise en compte d'objets dits mineurs ou secondaires lui ont valu le reproche infamant d'esthétisme ou d'essayisme $^{6}$. Ce qui ne l'empêche pas d'être à nouveau d'actualité, et ce, entre autre, parce qu'avec prémonition il avait reconnu «qu'il est tout à fait futile de vouloir réserver le titre de «science » à la détermination des lois et de ne pas l'accorder à l'observation des faits ${ }^{7} \gg$. On ne peut mieux dire la nécessité du constat ou de la description phénoménologique. C'est ce qui fait que ce que j'appellerai l'essai savant a sa place à l'Université, surtout en un temps où du fait du changement de valeurs qui s'opère, il est nécessaire d'être attentif à ce qui est in statu nascendi. Et pour ceux qui auraient quelque difficulté à l'admettre on peut rappeler que "gérer le savoir établi et sentir ce qui est en train de naître ne sont après tout que les deux pôles de la tension qui constituent l'harmonie conflictuelle de toute connaissance ${ }^{8} »$. Ainsi à côté d'une sociologie sociologisante une sociologie esthétisante peut jouer le rôle de tête chercheuse dans le nouveau monde qui s'annonce.

\footnotetext{
${ }^{4}$ R. A. Nisbet, la Tradition sociologique, Paris, PUF, 1984, p. 33 sq. Ainsi : «si j'insiste sur ce point c'est simplement parce que nous vivons à une époque où des enseignants de sociologie ... pleins d'éloquence et de bonnes intentions, insistent sur le fait que ce qu'il y a de scientifique... dans leur discipline résulte uniquement d'une réflexion procédant par définition puis résolution des problèmes ».

${ }_{6}^{5}$ Ibid., p. 35.

${ }^{6}$ Cf. R. Aron, la Sociologie allemande contemporaine, Paris, PUF, 1981, p.11. Également la préface de J. Freud in G. Simmel, Sociologie et épistémologie, Paris, PUF, 1981, p.11. «On a souvent reproché à Simmel d'avoir été un dilettante, brillant essayiste $\gg \ldots$

${ }^{7}$ G. Simmel, les Problèmes de la philosophie de l'histoire, Paris, PUF, 1984, p.164.

8 M. Maffesoli, la Connaissance ordinaire, Paris, Librairie des Méridiens, 1985, p. 29 sur le «formisme», ibid. p.98. Je m'y explique en particulier sur ce néologisme traduisant le terme « Formal » que Simmel applique à la sociologie.
} 


\section{II - LE « FORMISME » COMME CATÉGORIE COGNITIVE}

Ainsi dans le pluralisme de la connaissance peut exister une esthétisme méthodologique, celui-ci n'est en rien une abdication de la pensée. Qui plus est, on peut dire d'une manière heuristique, qu'il existe des moments où il est en parfaite congruence avec l'esprit du temps. C'est dans cette perspective que l'on peut parler d'une sociologie «formiste». Ce néologisme, sur lequel je me suis déjà expliqué, entend considérer la forme telle que G. Simmel en parle, comme un moule cognitif, et ce en s'inscrivant dans une tradition qui de la philosophie aristotélicienne à la biologie contemporaine, en passant par Goethe et Spengler, a ses lettres de noblesse.

Disons très rapidement que c'est parce que la réflexion esthétique fut une préoccupation constante chez Simmel qu'il élabora cette notion de forme ${ }^{9}$. Et celle-ci traduit une vraie obsession : celle de relation, de l'action réciproque, ou encore de ce qu'il appelle le « concept de rapport » (verhältnissbegriff).

La forme est avant tout un a priori qui ordonne les situations et les particularités observables. Elle met en relation des motivations et des manières d'être qui ne sont ni exclusivement rationnelles, ni exclusivement sensibles. Ainsi d'une manière nuancée et, dans le sens simple du terme, relativiste, le sociologue peut comprendre la complémentarité, peut-être faudrait-il dire la synergie des divers éléments du donné social. C'est à dessein que j'emploie ici cette expression. À l'encontre de la pensée critique qui, selon son étymologie, choisit, soupèse, sépare le vrai du faux, le formisme est plutôt une pensée affirmative : le donné est accepté parce qu'il est là. On voit bien ici l'influence nietzschéenne. Plutôt que de compromis ou d'acceptation du statu quo, on pourrait parler de la conjonction des sensibilités éléatique et héraclitéenne. Il est un texte de Simmel qui précise bien cette conjonction.

De même que notre connaissance saisit d'une part le durable dans le changement et d'autre part le changeant dans la durée, de même ... l'évolution organique constitue l'individu comme la somme des propriétés de l'espèce et lui octroie cependant la capacité de s'adapter à des formes et à des exigences nouvelles de la vie ${ }^{10}$.

\footnotetext{
${ }^{9}$ Dans son article « G. Simmel's Philosophy of Art and his sociological viewpoint » (Sociologica, vol. 5 $\mathrm{n}^{\circ}$ 2, Tokyo, 1981), Y. Atoji fait une bonne recension des écrits de Simmel sur l'art : depuis son traité «Psychologische und ethnologische studien über Musik (1882), jusqu'à «Der Konflit der modernen Kultur (1917). L'article de Y. Atoji paraître en traduction française dans le numéro de la revue Sociétés, Masson, Paris, consacré à Simmel.

${ }^{10}$ G. Simmel, Einleitung in die Moralwissenshaft ( $2^{\mathrm{e}}$ éd. tome I, p. 83, 1892), cit. in A. Mamelet, le Relativisme philosophique chez G. Simmel, F. Alcan, Paris, 1914.
} 
À priori et empirisme. Le donné est constitué à la fois par du pré-existant et par la cristallisation synthétique des attitudes induites par ce pré-existant; à la fois archétype et stéréotype. Il faudrait trouver des images dans la physique ou dans l'astrophysique contemporaines pour expliquer la dynamique des particules en mouvement et la statique de l'ensemble. C'est de cette tension qu'est constitué le donné social $^{11}$.

Tout l'art du sociologue sera de reconnaître, de constater et de décrire cette tension de la forme et du minuscule. Ainsi qu'il a été dit plus haut, moins critique que constat. Si une telle attitude contrevient à ce qui semble être, depuis l'Aufkärung, le fondement de la démarche intellectuelle, elle n'est pas moins performante lorsque l'on recommence à reconnaître que hors d'un lointain finalisme, les menus faits de l'existence quotidienne sont plein de sens en eux-mêmes : «Zwecklos aber sinnvoll ». On doit reconnaître que c'est le souci esthétique qui souligne l'importance de l'anodin, du détail comme constituant du tout. On rejoint là toute la réflexion de l'École de Palo Alto sur la «proxémie»; dans ses diverses modulations, le proche, ce que je peux toucher, ce qui se visualise directement n'est pas sans conséquences dans notre rapport au monde. Mais cela nécessite un changement d'optique. En effet, déclare Simmel, « notre inclinaison à critiquer est en connexion avec notre façon mécanique de voir les choses $^{12} »$.

Il me semble que tout l'intérêt du « formisme » est justement d'envisager un développement organique $\mathrm{du}$ social. La mise en relation et l'action réciproque (Wechselwirkung), dont il a été question, et qui s'appliquent à ces petites choses s'épuisant dans l'acte (repas, parure, coquetterie, rituels, politesse, etc.), font que la forme n'est nullement une Idée platonicienne, intangible et à l'effet parfaitement univoque. Bien au contraire, elle saisit le mouvant, l'interaction. C'est cela l'organicité. Pour reprendre l'image du développement (Entwicklung) employée au début, l'arbre croît et développe ses potentialités à partir de la «forme», ou encore pour user d'une autre métaphore tout comme la «déterminatio », la borne, qui délimitait un champ par rapport au désert, elle enclot mais donne à être ; elle permet qu'il y ait du blé plutôt que

\footnotetext{
${ }^{11}$ Sur le donné social cf. mon analyse, M. Maffesoli, La Violence totalitaire, Paris, PUF< 1979, p. 207.

${ }^{12}$ G. Simmel, Fragmente und Ajfsätze, Munich, Drei Masken Verlag, 1923, p. 28; trad. J. Freud, cit. Dans la Théorie de la forme chez Simmel, à paraître.
} 
rien. Permettant de penser le constant et le mouvant, la forme est une autre manière de rendre compte du principe interne d'organisation, de la « ratio seminalis » qui fait que dans toute structuration il y ait correspondance, action, retro-action, toute chose que nous a appris la cybernétique. C'est en ce sens que l'esthétisme de la forme peut être appelé un moule cognitif. Toutes les données de base «ont déjà été modelées par des formes a priori ${ }^{13} \gg$ un peu comme des informations codées qu'il suffit de savoir lire.

Ainsi donc même si d'une part cela ne permet pas d'élaborer des lois générales, d'autre part de raisonner en terme de « devoir être », le formisme permet au sens strict du terme de cohérer les éléments hétérogènes qui constituent nos sociétés complexes. On peut préciser, in fine, que cette cohérence utilise le vecteur de ce que H. Corbin et G. Durand appellent la fonction imaginale. En effet, sans qu'il soit possible de s'y étendre, on peut émettre l'hypothèse qu'après la prévalence d'une pensée iconoclaste - méfiance vis-à-vis de l'imaginaire, recherche de l'authenticité, procédure de soupçon et donc de la critique - l'on assiste à une revalorisation des modes d'apparaitre. Dans la logique du donné accepté « parce qu'il est là » et dans le cadre de la description qui est corrélative de cette acceptation, la prise en compte de l'apparence pourra être un levier méthodologique non négligeable. Et il est flagrant que ce qui n'était qu'une intuition au début de ce siècle devient parfaitement pertinent en un moment où l'image, publicitaire télévisuelle, électronique, se capillarise dans l'ensemble du corps social. Nous avons à prendre en compte les effets de cette diffusion sous peine de ne rien comprendre à notre quotidien. Si pour reprendre une hypothèse formulée au début, le Verbe, et son pendant l'Image, prévalent sur l'Action, une sociologie de l'imaginaire social ne sera pas un simple supplément d'âme philosophique, mais bien le cœur de la démarche réflexive.

Suivant le balancier des histoires humaines, il s'agit là d'une accentuation qui a déjà existé. Pour ne prendre qu'un exemple, Peter Brown, avec une grande originalité, montre que l'on peut comprendre l'Antiquité tardive à partir de ce qu'il appelle le «style» des échanges sociaux, i.e. le style des rapports au divin, le style des relations quotidiennes. Ce qui fait du style une catégorie du savoir ${ }^{14}$.Qu'est-ce à dire sinon que la vie sociale ne se réduit pas à l'ordre de la causalité rationnelle, ou ne peut être subsumée sous une loi, ou un ensemble de lois universelles. Il est donc des moments où cette

\footnotetext{
${ }^{13}$ G. Simmel, les Problèmes de la philosophie de l'histoire, op. cit., p.83.

${ }^{14} \mathrm{P}$. Brown, Genèse de l'Antiquité tardive, Paris, Gallimard, 1983, p. 16 sq. et la préface de P. Veyne qui fait état du style comme catégorie du savoir. Sur l'apparence et la théâtralité je renvoie à mes analyses, M. Maffesoli, la Conquête du Présent, Paris, PUF, 1979, ch. VII et VIII.
} 
particularité revient sur le devant de la scène, où l'homme ne s'inscrira pas dans une finalité orientée, poussé par des causes précises, mais où d'une manière stochastique il va vivre au présent des situations ponctuelles et éphémères. Ce qui privilégie la manière dont ces situations s'expriment, se donnent à voir, accentuent l'apparence.

Il s'agit là de périodes où dominent les sentiments de finitude et de tragique; et le «formisme » en ce sens n'est qu'une mise en perspective de la précarité des choses. Il est une remarque de Simmel, aux accents bien nietzschéens qui indique que l'attitude « de l'artiste repose... sur le présupposé selon lequel la signification profonde des choses se révèle adéquatement dans leur apparence... ${ }^{15} \gg$ Cette liaison de la profondeur et de la surface (qui n'est pas sans rappeler celle du Pont et de la Porte, Brücke und Tür) est instructive pour apprécier, d'une manière non normative, l'efflorescente bigarrure du spectacle quotidien que l'on peut observer dans les places et les rues des mégapoles modernes. Ainsi que pour une coupe histologique, étudier une société à partir d'une description de sa peau, voilà l'enjeu d'une sociologie « formiste ». Pour celle-ci il n'y a pas une vraie existence derrière celle qui se donne à voir, elle se contente de prendre au sérieux les divers styles de vie constitutifs du « theatrum mundi ».

\section{III - LA SOCIALITÉ MICROSCOPIQUE}

Excéder l'évaluation. Tâche urgente et pourtant bien difficile. Il faut bien reconnaître que la perspective esthétique et le formisme qui en est l'expression ne sont pas chose aisées pour le sociologue. On sait en effet que pour ce dernier, je cite ici Nisbet, depuis le XVIII ${ }^{\mathrm{e}}$ siècle la politique «devient ... un mode de vie intellectuel et moral ${ }^{16} \gg$. Ainsi, dans son orientation générale comme dans ses modalités spécifiques, l'être-ensemble ne pourrait être compris que sur l'horizon du politique, qui se résume assez bien dans l'attitude projective : l'action, le futur, le lointain. Face à cela, le paradigme esthétique qui se met en place peut paraître quelque peu inactuel qu'il serve de révélateur aux diverses expressions de la socialité contemporaine. Celles-ci ne manquent pas d'être anomiques pour ce qui regarde l'injonction morale de la politique. Mais peut-être s'agit-il de ce que j'ai appelé un «immoralisme éthique », c'est-à-dire

\footnotetext{
${ }^{15}$ Cité par R. A. Nisbet, op.cit., p. 272. Sur la stylisation de l'existence, cf. G. Simmel, Mélange de philosophie relativiste, Paris, Felix Alcan, 1912, p. 217, et les Problèmes de la philosophie de l'histoire, op.cit. ; p. 53. Voir aussi son texte sur la coquetterie, dans Sociétés, $\mathrm{n}^{\circ} 3$, p. 20 sq.

${ }^{16}$ R. A. Nisbet, la Tradition sociologique, op.cit., p.54.
} 
porteur d'une moralité qui est créée collectivement et non point imposée d'une manière surplombante $^{17}$. En effet le propre de l'«aisthesis » est de reposer sur une expérience partagée. En simplifiant à l'extrême on peut rappeler que tout comme le rationalisme, dans l'ordre de la pensée, tend à séparer, à discriminer, pour analyser, de même il est cause et effet de l'atomisation, faut-il dire de l'individualisme dans la vie sociale. Dans l'un et dans l'autre cas, la base étant le «principium individuationis ». L'ayant traité abondamment (Violence totalitaire, chap. IV, L'ombre de Dionysos : Introduction et chap. I), je ne reviendrai pas sur cette question.

Il faut par contre insister sur le fait que l'«aisthesis » favorise l'interaction. L'historien de l'art W. Worringer parle de certaines périodes où prédomine la procédure « tactile», on peut dire métaphoriquement que pour le paradigme esthétique qui nous concerne il s'agit bien du «toucher », du sensible, ou encore comme cela a été très bien dit, de «l'attraction sociale ${ }^{18} »$. Plus qu'une addition d'individus contractuellement liés, schéma qui prévaut à partir des Lumières, on peut imaginer qu'il existe, d'une manière quelque peu mystérieuse, une conjugaison des sensibilités, ce qui permettrait de comprendre la multiplication des réseaux sociaux et des petits groupes qui les constituent. Néo-tribalisme versus Société. Un certain nombre de sociologues tels A. Schutz, P. Berger et T. Luckmann (malheureusement peu connus ou utilisés in pectore, en France) ont insisté sur l'importance de la relation, de l'expérience interactive pour la réalité sociale ${ }^{19}$. C'est en s'inspirant d'eux que H. R. Jauss (initiateur de ce qui s'appelle l'École de Constance) a souligné le lien existant entre l'interaction et l'expérience ou la jouissance esthétique. Il faudra y revenir, mais à la différence de la réflexion sur l'art de l'École de Francfort, Adorno en particulier, qui repose sur la critique de la jouissance comme catégorie bourgeoise, l'École de Constance montre que le sensible, l'intersubjectivité, tel un fil rouge parcourt le corps social et lui assure la perdurance que l'on sait.

Ainsi à partir des catégories de hic et nunc, Jauss montre comment « la réalité quotidienne est éprouvée comme celle d'un monde intersubjectif que je partage avec

\footnotetext{
${ }^{17}$ Sur « l'immoralisme éthique », cf. M. Maffesoli, l'Ombre de Dionysos, contribution à une sociologie de l'orgie, Paris, éd. Librairie des Méridiens, $2^{\mathrm{e}}$ éd., 1985.

${ }^{18} \mathrm{Je}$ renvoie ici à l'excellente et érudite étude de P. Tacussel : l'Attraction sociale (la dynamique de l'Imaginaire dans la société monocéphale), Paris, Lib. des Méridiens, 1984, cf. également W. Worringer, Abstraction et Einfühlung, Paris, Klincksieck, 1978.

${ }^{19} \mathrm{Cf}$. en particulier C. Javeau «La sociologie du quotidien : paradigmes et enjeux », dans la Revue suisse de sociologie, 1, 1983, pp. 21-36.
} 
d'autres ». L'« Einfühlung » est à la fois jouissance esthétique et processus empathique qui me lient aux autres ${ }^{20}$. Il faut reconnaître que l'on est en présence d'un certain vitalisme, qui régulièrement renaît dans les structurations sociales, et donc dans les constructions intellectuelles. En effet, il y a toujours une liaison entre l'esthétique et les diverses manifestations de la vie. C'est particulièrement flagrant pour Simmel qui tint constamment les deux bouts de la chaîne ${ }^{21}$. Et ce à partir de deux angles d'attaque ; d'une part celui de la forme qui est, on l'a vu, relation, action réciproque, " concept de rapport ", d'autre part celui de la dimension symbolique qu'il convient de comprendre non pas comme une simple catégorie psychologique mais bien, suivent en cela son étymologie, comme vecteur d'intense communication. Il parle même de système symbolique permettant de saisir le «tissus de faits vécus ${ }^{22}{ }$.

Il est nécessaire d'insister là-dessus, ne serait-ce que pour relativiser, sinon désamorcer tout à fait, l'adéquation que l'on fait couramment entre l'accentuation du quotidien et de l'esthétique d'une part et celle de l'individualisme d'autre part. Pour ma part, ainsi que je l'ai indiqué, je considère que l'individualisme est étroitement lié à l'ordre du politique. Dans une remarque qui mérite attention, Simmel fait un parallèle entre la problématique individualiste et celle du matérialisme historique, elles vont considérer «les intérêts communs à un ensemble d'individus »; le général étant pour elles «ce à quoi tous sont parties prenantes plutôt que ce qui est commun à tous ${ }^{23}$ ». Il s'agit d'une différence qualitative, d'un côté l'accent est mis sur le monade et son pendant l'association rationnelle, de l'autre il est plutôt sur le groupe et son « ciment» affectif. En d'autres termes la Politique versus la Socialité. Faut-il suivre Nisbet lorsqu'il déclare d'une manière péremptoire que «Simmel récuse l'individualisme analytique ou utilitariste ... même ... s'il s'exprime en des termes qui peuvent parfois laisser croire à tort que son œuvre se rattache à l'individualisme ${ }^{24} \gg$ ? Ce n'est pas certain. En effet Simmel est sur ce point très partagé : en homme de son temps, nombre de ses analyses renvoient au politique (i.e. à l'individualisme), mais en même temps il

\footnotetext{
${ }^{20}$ Cf. H. R. Jauss, Pour une esthétique de la réception, Paris, Gallimard, 1978, pp. 292, p. 278, 149 ; P. Berger et T. Luckmann, La Construction sociale de la réalité, Paris, Lib. des Méridiens, 1986.

${ }^{21}$ Cf. l'article de Y. Atoji, «G. Simmel's Philosophy of Art and his Sociological Viewpoint », op. cit., p. 1 qui explique bien cette liaison esthétique-vie: «his philosophy of art... is closely related to his philosophy of life (Lebensphilosophie) ».

${ }^{22}$ G. Simmel, les Problèmes de la philosophie de l'histoire, op. cit., p. 111.

${ }^{23}$ G. Simmel, ibid., p. 131.

${ }^{24}$ R. A. Nisbet, la Tradition sociologique, op.cit., pp. 128, 127.
} 
ne peut pas ne pas voir la multiplication des micro-groupes qui, quoiqu'à l'état naissant, se diffusent dans les métropoles modernes.

C'est ce que reconnaît Nisbet en remarquant que Simmel «s'intéresse tout autant aux processus imperceptibles qu'aux structures ».

Ce qui est certain, dans l'optique que je défens ici, c'est qu'il manifeste une réelle attention aux communautés moléculaires » qu'il est fasciné par les relations microscopiques, et que ses analyses des sociétés secrètes, des interactions des dyades et des triades (Nisbet, p. 127), permettent dans le cadre formiste d'observer le grouillement du bouillon de culture sociétal. En bref on peut dire que le minuscule n'est pas réductible à l'individu, alors que celui-ci se complaît dans sa solitude, et peut tout au plus l'associer à d'autres (Politique), celui-là ne se comprend qu'en relation, il est cause et effet du cadre, de la forme qui le fait ressortir (socialité) ${ }^{25}$.

\section{IV - L'INTERSUBJECTIVITÉ ESTHÉTIQUE}

C'est ce sentiment partagé dans la socialité, cette expérience commune, qui en appellent à l'empathie, à l'intuition du sociologue. Il faut d'une certaine manière qu'il sache voir de l'intérieur. Art de faire et art de dire. C'est à cette condition que le sociologue pourra être le mieux à dire ce qu'il en est de la rhétorique sociale. C'est une question d'implication en quelque sorte.

Proust disait que l'on ne peut comprendre un livre si l'on n'est pas capable « d'en mûrir lentement les équivalents dans son propre cœur ». Peut-être faut-il dire la même chose pour le sociologie : est-il à même de comprendre ce monde s'il ne peut en lui-même l'éprouver comme microcosme; ou à tout le moins entrer en correspondance avec certains éléments de ce monde qu'il entend analyser. On peut d'ailleurs postuler qu'une certaine dose d'identification est cela même qui permet la «typification » ou l'établissement de typologies. En tout cas il s'agit d'une perspective que nombre de chercheurs ne rejettent plus à priori, tant il est vrai qu'une subjectivité affirmée est plus facilement maîtrisée que celle qui, tout en ne s'avouant pas comme telle, n'en est pas

\footnotetext{
${ }^{25}$ Cf. par exemple G. Simmel, les Problèmes de la philosophie de l'histoire, op. cit., « en recherchant les similarités (l'historien) soumet son matériau à une interprétation symbolique, alors qu'une méthodologie individualiste du type de celle qu'adoptent les sciences de la nature chercherait à réduire ce matériau à ses composantes élémentaires pour le soumettre à des lois » (p. 169).
} 
moins présente - sous forme de valeurs, de préjugés, de convictions - dans les livres les plus objectivistes.

À cet égard G. Simmel peut orienter notre réflexion en deux directions que je donne en conclusion comme pistes de recherche.

Tout d'abord, le sens artistique, «l'aisthesis» comme mécanisme de connivence, peut-être même de complicité. «Être capable d'empathie, c'est-à-dire être capable de retrouver les motivations des acteurs... c'est pourquoi on dit quelquefois que l'historien est et doit être un artiste ${ }^{26}$. C'est banalité de le dire, tant il est vrai que le choix de nos objets d'étude est fortement conditionné par nos affects, soient-ils rationalistes. Ce que l'on peut tout de même rajouter c'est que le sens artistique, en référence à notre développement, ne doit pas se porter uniquement sur le contenu, mais aussi sur le contenant. Il y a donc là «projection de notre propre moi » (p. 127) qui permet la reproduction d'expériences qui n'ont pas été vécues. Cette projection est naturellement le fait du «génie »: artiste stricto sensu, intellectuel ou divers créatifs ; elle est également le fait de l'homme du commun. D'où l'importance qu'il convient d'attribuer à la banalité dans la vie sociale. En prenant soin de faire remarquer que cette banalité est avant tout lieu d'expériences communes.

L'autre direction est celle que j'ai désignée sous le terme mystique de « microcosme », mais qu'en référence à Schutz on peut pointer comme étant celle de la «typicalité ». D'autre part elle accentue les singularités causales, d'autre part elle relève les similitudes et les ressemblances. Ainsi chaque individu peut être considéré comme un en soi. En tant que tel significatif, mais dont justement la signification ne vaut que dans la co-relation, la co-respondance. À la manière dont Max Weber parle du pluricausalisme, la typicalité nous renvoie à un problème de «constellations ». Dans un monde complexe, cahotique, contradictoriel, il s'agit de faire des regroupements d'ensembles. On ne réduit pas, conceptuellement, une vie multiforme, mais on repère des situations, de la plus anecdotique à la plus importante, que l'on essaie d'imputer à des formes spécifiques.

Il me semble que le paradigme esthétique, ici esquissé, est la démarche la moins mauvaise pour appréhender les processus de communication, de sympathie, d'identification, d'association qui de plus en plus structurent nos sociétés. J'ai proposé d'appeler cela, le jeu de la passion. Nombre d'adhérences télévisuelles, de fascinations

\footnotetext{
${ }^{26}$ G. Simmel, les Problèmes de la philosophie de l'histoire, op.cit., p. 121.
} 
fictionnelles ou même d'effervescences politiques sont sans cela incompréhensibles : du corps mystique qui chaque semaine se crée autour de Dallas à l'afoulement des grands magasins ou autres rassemblements sportifs, on retrouve une interaction affective qui se moque bien de nos jugements de valeur, et qui réclame une mise en forme faite de lucidité et d'humilité. Pour Berger et Luckmann la «réalité de la vie quotidienne se présente comme un mode intersubjectif, un monde que je partage » et cette réalité leur paraît être «souveraine $»^{27}$. Il s'agit là d'une remarque de bon sens que les sociologues ne peuvent plus rejeter comme «spontanée » et donc comme idéologique. Mais elle nécessite une conversion de l'esprit qui fasse de nous non plus des critiques, des contempteurs mais bien des esthètes de l'existence ${ }^{28}$. Et ce non pas pour se complaire dans un narcissisme ou un individualisme qui n'est plus de mise, mais au contraire - si on suit encore Simmel lorsqu'il décrit cette activité esthétique - pour se détacher «du moi pour se dissoudre dans l'objet, qu'aucune dualité ne ... sépare plus de cet objet $»^{29}$.

Ainsi l'intuition, cette vue du dedans, peut devenir levier méthodologique, et fait que l'intellectuel, cynique vis-à-vis de tous les pouvoirs, peut rester le gardien de la parole. Et l'on sait que c'est la circulation de cette dernière qui fonde et conforte la socialité.

Recebido em : 28/03/2015

Aprovado em : 25/05/2015

\footnotetext{
${ }^{27}$ P. Berger et T. Luckmann, La Construction sociale de la réalité, op. cit., p. 23 sq.

${ }^{28}$ À cet égard il faut savoir tirer les conséquences de ce que L. Dumont appelle la «croissance considérable de la catégorie esthétique », et peut-être ne plus avoir peur « de spéculer, voire divaguer » (cf. Homo Aequalis, Paris Gallimard, 1977. p. 247, note 12). En extrapolant quelque peu la catégorie esthétique, c'est-à-dire en ne considérant pas uniquement les grandes œuvres de la culture, on peut imaginer que l'ensemble de la vie courante devient une ouvre d'art.

${ }^{29} \mathrm{G}$. Simmel, les Problèmes de la philosophie de l'histoire, op. cit., p. 122.
} 